antibody to $\mathrm{EB}$ virus. This suggests that at least some of the patients in whom the Paul-Bunnell reaction is negative are cases of " true " glandular fever. The remaining patients might have been suffering from a different disease or diseases - possibly due to cytomegaloviruses ${ }^{9}{ }^{10}$ - which are known to cause glandular-fever-like syndromes.

These results support the view that EB virus is the cause of glandular fever, but other evidence suggests that the ecology of the virus in human populations is far from being understood. For example, EB virus has been detected in cultures of leucocytes from patients with glandular fever ${ }^{11}$ - though not until cultures had been maintained in vitro for two to four weeks. But it has also been found in cultures from patients with various forms of malignant diseases ${ }^{12}{ }^{13}$ and even in cells from two healthy adults. ${ }^{14}$ Leucocytes from patients who had had glandular fever previously ${ }^{11}$ also contained the virus, suggesting that it may persist in the lymphoid cells after the initial infection. Persistence of virus in host cells in spite of circulating antibody in the serum is known to occur with two other herpesviruses-herpes simplex and varicella-zoster viruses. Infection with $\mathrm{EB}$ virus is clearly widespread: $50 \%$ of 4-year-old children have antibody to the virus, and the incidence of antibody rises to more than $80 \%$ of people over 70 years of age. ${ }^{15}$ It seems, then, that $\mathrm{EB}$ virus resembles herpes simplex and cytomegaloviruses in causing frequent but usually symptomless infections, with occasional production of disease in an especially susceptible host.

Some doubts remain. How specific are serological reactions with a virus which cannot be freed from the cells in which it is growing or subcultured in other cells by the usual techniques? Further uncertainties are raised by the electron microscopic studies of $\mathrm{H}$. L. Moses and his colleagues ${ }^{16}$ on lymphoid cell lines established from patients with glandular fever. They found that the presence of much smaller viruslike particles was a more consistent finding in the cells than the larger EB virus particles. Until these doubts are resolved we cannot be certain that EB virus is the cause rather than merely a "passenger" virus in glandular fever. Its relationship to Burkitt's lymphoma remains tantalizingly unknown.

\section{Bone and Joint Changes in Haemochromatosis}

Since H. R. Schumacher ${ }^{1}$ described in 1964 a specific arthropathy in idiopathic haemochromatosis there has been considerable interest throughout the world in this unexpected complication, for so long overlooked. The arthropathy is often associated with the characteristic radiological appearance of chondrocalcinosis. This last condition is due to deposition of calcium pyrophosphate. It is most often seen in the menisci of the knee, in the articular hyaline cartilage of the larger joints, and in the fibrocartilage of the symphysis pubis and the triangular ligament of the wrist.

Recently E. N. Wardle and J. T. Patton ${ }^{2}$ have described the bone and joint changes in 14 patients with haemochromatosis. The authors emphasize that bony swellings of the hands are common, particularly of the metacarpophalangeal and proximal interphalangeal joints. Radiographs show small cysts, irregularity of the articular surface, sclerosis, and loss of joint space. Similar changes are present in the carpal bones and in the inferior radio-ulnar joints. Symptoms may be minimal. Other patients complain of pain and stiffness in the hands, and if the larger joints are affected (in about a third of the patients) considerable pain and disability can result. The radiological changes in larger joints are similar, with erosion of cartilage and sclerosis of underlying bone. Osteophytes are not common, but the changes, particularly in the hip joints, may be indistinguishable from osteoarthritis.

Superimposed on this chronic arthropathy there may be acute attacks of inflammatory synovitis. These occurred in 8 out of 16 patients described by E. Hamilton and colleagues ${ }^{3}$ and are due to liberation of calcium pyrophosphate crystals into the joint fluid. Crystal synovitis also occurs in the other conditions in which chondrocalcinosis is found-namely, hyperparathyroidism, gout, and idiopathic chondrocalcinosis (the "pseudo-gout syndrome"). ${ }^{4}$ Chrondrocalcinosis was present in 3 out of 13 patients in Wardle and Patton's series, ${ }^{2}$ and in 10 out of 16 with arthropathy described by Hamilton and colleagues. ${ }^{3}$ It is probably secondary to the deposition of calcium pyrophosphate in cartilage damaged by some other process. Under normal conditions pyrophosphate is hydrolysed to soluble orthophosphate by the pyrophosphatase enzyme present in cartilage. ${ }^{5}$ The excess iron in the joint tissues in haemochromatosis may inhibit pyrophosphatase activity and permit depositions of pyrophosphate to occur. However, inhibition of pyrophosphatase is not a specific consequence of excess of iron, since chondrocalcinosis occurs in the other conditions mentioned.

The initial damage to the joints in haemochromatosis presumably results from the deposits of iron, though the exact relationship between tissue damage and iron deposition is uncertain. Articular cartilage is known to be dependent on the synovium for its nutrition, and in $1935 \mathrm{~J}$. H. Sheldon in his famous monograph ${ }^{6}$ described haemosiderin deposits in the synovium and in the articular cartilage. In more recent cases which have been examined either at necropsy or by biopsy iron has been noted more frequently in the synovium than in the articular cartilage. In some cases deposits are still found in the synovium after treatment by venesection. Certainly the arthritis can develop after venesection has been completed, and unfortunately venesection does not lead to improvement in the arthropathy. Indeed, progressive deterioration may be observed, though the patient in other respects is improved. ${ }^{3}$ Perhaps damage to the articular cartilage, once induced, is irreversible. This is true of other consequences of haemochromatosis, including cirrhosis of the liver. Removal of excess iron from the tissues, though it may lead to improvement in liver function, does not alter the fibrosis and nodular regeneration. ${ }^{7}$ In general, patients with haemochromatosis who develop arthritis are older and have had the disease longer than those without arthritis.

1 Schumacher, H. R., Arthritis and Rheumatism, 1964, 7, 41.

Wardle, E. N., and Patton, J. T., Annals of Rheumatic Diseases, 1969, 28,15 .

Hamilton, E., Williams, R., Barlow, K. A., and Smith, P. M., Quarterly fournal of Medicine, 1968, 37, 171.

- Moskowitz, R. W., and Katz, D., American fournal of Medicine, 1967,

43, 322.
McCarty, D. J., Hogan, J. M., Gatter, R. A., and Grossman, M., fournal of Bone and foint Surgery, 1966, 48-A, 309.

Sheldon, J. H., 1935, Haemochromatosis. London, Oxford University

Press.
Williams R., Smith, P. M., Spicer, E. J., Barry, M., and Sherlock, S., Quarterly fournal of Medicine, 1969, 38, 149.

- Muirden, K. D., and Senator, G. B., Annals of Rheumatic Diseases, Muirden, K. D.

- Delbarre, F., Semaine des Hôpiteaux de Paris, 1960, 36, 3279.

${ }^{10}$ Seftel, H. C., et al., British Medical fournal, 1966, 1, 642. 
Concentrations of iron in the joint tissues as high as occur in haemochromatosis are also found in rheumatoid arthritis, though it is believed here that the deposits arise from the continual oozing of blood from vascular granulation tissue. ${ }^{8}$ The iron lies deep in the synovium, which shows proliferation and infiltration by inflammatory cells, whereas in haemochromatosis the deposits of iron are found in the cells of the synovial lining and the underlying stroma appears undamaged.

Osteoporosis may also occur. Schumacher ${ }^{1}$ found it in the spine in 6 out of 23 patients and F. Delbarre ${ }^{9}$ described some severe cases. However, Wardle and Patton ${ }^{2}$ noted osteoporosis in only 2 of 13 spines examined, though localized osteoporosis of the hands was evident in 7 . Two of their patients had hypercalciuria, but the exact cause of the osteoporosis is uncertain. H. C. Seftel and colleagues ${ }^{10}$ from South Africa have described spinal osteoporosis in the Bantu in association with severe siderosis. The siderosis and osteoporosis were also closely associated with deficiency of ascorbic acid, and they postulated that it was the latter which led to the diminished formation of bone. The large storage deposits of iron, which are in the ferric form, represent a massive oxidation potential, and part of the available ascorbic acid may be irreversibly oxidized by these deposits. A contributing factor in the Bantu is deficient dietary intake, and similar though less severe changes have been noted in idiopathic haemochromatosis and transfusional siderosis. ${ }^{11}$ Another important factor in the osteoporosis may be deficiency of androgen. Hypogonadism is a well-known feature of haemochromatosis, and Wardle and Patton found low levels of urinary 17oxosteroid and 17-oxogenic steroids in their patients.

Since venesection will not improve the arthropathy, and since prevention appears unlikely, what can be done to help these patients? In those with relatively minor symptoms aspirin or one of the anti-inflammatory agents such as phenylbutazone or indomethacin can be of considerable help. Acute attacks of arthritis due to synovitis require immobilization of the joint with aspiration of the joint fluid. This often gives relief as well as enabling confirmation of the diagnosis by examination of the joint fluid by polarized-light microscopy. Severe painful arthritis of the hip can be helped by an osteotomy and other surgical procedures.

\section{Motor Neurone Disease and Neuronal Metabolism}

The aetiology of motor neurone disease is unknown. Whether the disease presents clinically with progressive wasting of voluntary muscles in the limbs (progressive muscular atrophy), with less evident muscular atrophy and fasciculation but with predominant signs of degeneration in the corticospinal tracts (amyotrophic lateral sclerosis), or whether the motor nuclei of the cranial nerves and the corticospinal tracts in the brain stem are predominantly involved (progressive bulbar palsy), there is little or no evidence to indicate the nature of the disease process in the upper or lower motor neurones which accounts for or accompanies their degeneration.

One obstacle to studies of neuronal metabolism in this disease is that, whereas in primary muscle disease the metabolism of the muscle fibre can be studied by biopsy, this cannot be used for studying changes in the anterior horn cells of the spinal cord. The recent description of a genetically determined form of lower motor neurone degeneration in the mouse ${ }^{1}$ may mean that an animal has been found on which biochemical and electrophysiological studies may be possible in the future. But it is uncertain whether this mouse disease is strictly comparable with the human disorder.

In 1957 E. W. Poole ${ }^{2}$ reported that ischaemic and postischaemic paraesthesiae were absent in the limbs of patients with motor neurone disease even after periods of arterial occlusion which constantly produced such phenomena in normal persons. It is a well-known clinical finding that patients with motor neurone disease show no subjective or objective sensory abnormality, so that Poole's ${ }^{2}$ findings were difficult to explain. B. Shahani and W. Ritchie Russell ${ }^{3}$ have now reinvestigated this phenomenon in three patients suffering from motor neurone disease and in three control persons. They were stimulated to do so by the findings of G. Gregerson $^{4}$ and K. N. Seneviratne and O. Peiris, ${ }^{5}$ who showed that peripheral nerves in cases of diabetes may be unusually resistant to ischaemia. They have studied compound motor action potentials ( $M$-waves) recorded from the abductor pollicis brevis muscle during supramaximal stimulation of the median nerve and $\mathrm{H}$-waves (reflex responses obtained during submaximal stimulation), as well as $M$-waves recorded from one soleus during stimulation of the medial popliteal nerve. These observations were carried out in their cases and in controls before and after arterial occlusion produced by the application of a sphygmomanometer cuff to the upper arm or thigh at a pressure greater than the patient's systolic arterial blood pressure for periods of 15-20 minutes. The authors have confirmed that ischaemic and postischaemic paraesthesiae are either absent or mild in patients with motor neurone disease, and from the results of their observations they have concluded that the absence of such paraesthesiae in this condition can be related to a relatively high resistance of large sensory nerve fibres to ischaemia. They conclude that this abnormality of peripheral nerves may indicate that in motor neurone disease neuronal metabolism is altered.

These preliminary results need to be confirmed in studies of larger groups of patients. Moreover, it should be possible to show that sensory fibres are abnormally resistant to ischaemia by methods utilizing direct stimulation and recording from them. It is important too that the sensitivity of the motor nerve fibres to ischaemia should be studied in patients with motor neurone disease; inspection of the $M$-waves illustrated by Shahani and Ritchie Russell ${ }^{3}$ suggests that the sensitivity of these fibres in patients was probably similar to that in control subjects. Hence, though these observations are of interest, and though studies of the behaviour of peripheral nerve fibres in patients with motor neurone disease may perhaps allow us to draw valid conclusions about the behaviour of the whole neurone, the finding that sensory nerve fibres in this condition are abnormally resistant to ischaemia can be regarded as no more than a first step along a road beset with many difficulties.

1 Duchen, L. W., and Strich, S. J., Fournal of Neurology, Neurosurgery and Psychiatry, 1968, 31, 535.

2 Poole, E. W., Fournal of Neurology, Neurosurgery and Psychiatry, 1957, 20, 225.

- Shahani, B., and Russell, W. R., Fournal of Neurology, Neurosurgery and Psychiatry, 1969, 32, 1.

- Gregerson, G., fournal of Neurology, Neurosurgery and Psychiatry, 1968, 31, 175 .

- Seneviratne, K. N., and Peiris, O. A., Fournal of Neurology, Neurosurgery and Psychiatry, 1968, 31, 338. 